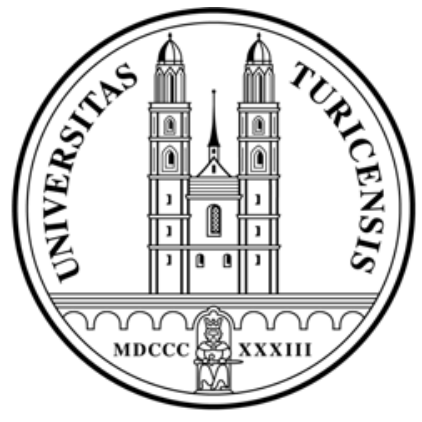

Institute for Empirical Research in Economics

University of Zurich

Working Paper Series

ISSN 1424-0459

Working Paper No. 364

Probabilistic Choice and Stochastic Dominance

Pavlo R. Blavatskyy

April 2008 


\title{
Probabilistic Choice and Stochastic Dominance
}

\author{
Pavlo R. Blavatskyy ${ }^{\dagger}$ \\ Institute for Empirical Research in Economics \\ University of Zurich \\ Winterthurerstrasse 30 \\ CH-8006 Zurich, Switzerland \\ Phone: $+41(1) 6343586$ \\ Fax: $+41(1) 6344978$ \\ e-mail: pavlo.blavatskyy@,iew.unizh.ch
}

April 2008

\begin{abstract}
This paper presents an axiomatic model of probabilistic choice under risk. In this model, when it comes to choosing one lottery over another, each alternative has a chance of being selected, unless one lottery stochastically dominates the other. An individual behaves as if he compares lotteries to a reference lottery-a least upper bound or a greatest lower bound in terms of weak dominance. The proposed model is compatible with several well-known violations of expected utility theory such as the common ratio effect and the violations of the betweenness. Necessary and sufficient conditions for the proposed model are completeness, weak stochastic transitivity, continuity, common consequence independence, outcome monotonicity, and odds ratio independence.
\end{abstract}

Keywords: probabilistic choice, first-order stochastic dominance, expected utility theory, random utility model, risk

JEL Classification codes: C91, D81

\footnotetext{
${ }^{\dagger}$ I am grateful to Christian Ewerhart, Michael Kosfeld, Wolfgang Köhler, Tony Marley and Ganna Pogrebna as well as the participants at research seminar at the University of Zürich (January 25, 2007) for helpful comments.
} 


\section{Probabilistic Choice and Stochastic Dominance I. Introduction}

The aim of this paper is to present a simple model of decision making under risk that captures two stylized facts: 1) in general, people choose probabilistically between risky lotteries; 2) people seldom violate strict dominance or transparent first-order stochastic dominance. Numerous experimental studies of repeated decision making under risk demonstrate that individual choices are probabilistic in nature (e.g. Camerer, 1989; Starmer and Sugden, 1989; Hey and Orme, 1994; Wu, 1994; Ballinger and Wilcox, 1997; Loomes and Sugden, 1998; Hey, 2001; Schmidt and Hey, 2004; Schmidt and Neugebauer, 2007).

For example, consider a choice between getting $£ 10$ for certain and a $50 \%-50 \%$ chance of receiving either $£ 30$ or nothing. When facing this decision problem repeatedly, some people opt for the sure amount on one occasion and for the risky lottery on the other. Loomes and Sugden (1998) observe that 11 out of 46 subjects $(23.9 \%)$ reverse their initial choice on the second repetition of this decision problem. Traditional decision theories (e.g. expected utility theory and cumulative prospect theory) interpret such contradictory choice pattern as a sign of indifference between the sure amount and the risky lottery.

Consider now a choice between $£ 10$ for certain and a risky lottery that yields a $60 \%$ chance of $£ 30$ (zero otherwise). If an individual is genuinely indifferent between $£ 10$ for sure and a $50 \%$ chance of $£ 30$, he strictly prefers a $60 \%$ chance of $£ 30$ over $£ 10$ for sure. However, Loomes and Sugden (1998) find that 9 out of 46 subjects (19.6\%) choose $£ 10$ for sure over a $50 \%$ chance of $£ 30$ and vice versa and they also choose $£ 10$ for sure over a $60 \%$ chance of $£ 30$ on at least one occasion. Traditional decision theories cannot explain this 
empirical finding because an individual cannot be simultaneously indifferent between $£ 10$ for sure, a $50 \%$ chance of $£ 30$ and a $60 \%$ chance of $£ 30$.

Even though people choose probabilistically between $£ 10$ for sure and a $50 \%$ chance of $£ 30$ as well as between $£ 10$ for sure and a $60 \%$ chance of $£ 30$, they do not choose probabilistically between a $50 \%$ chance of $£ 30$ and a $60 \%$ chance of $£ 30$. People seldom violate strict dominance or transparent first-order stochastic dominance (e.g. Carbone and Hey, 1995; Loomes and Sugden, 1998; Hey, 2001). For example, Loomes and Sugden (1998) find that only one out of 46 subjects (2.2\%) has chosen a $15 \%$ chance of $£ 30$ over a $20 \%$ chance of $£ 30$ (zero otherwise) on one of two repetitions of the decision problem.

Popular models of probabilistic choice such as the Fechner model of random errors (Fechner, 1860; Hey and Orme, 1994; Hey, 1995; Buschena and Zilberman, 2000), the Luce choice model (Luce and Suppes, 1965; Camerer and Ho, 1994; Wu and Gonzalez, 1996), and the constant error/tremble model (Harless and Camerer, 1994; Carbone, 1997; Loomes et al., 2002) generally predict too many violations of strict dominance and transparent stochastic dominance. Blavatskyy (2007) develops a model of probabilistic choice (stochastic expected utility theory) that rules out violations of strict dominance but allows for violations of stochastic dominance. Blavatskyy (2006b) extends this model to discriminate between low rates of violation when stochastic dominance is transparent and high rates of violation when stochastic dominance is not transparent. Fishburn (1978) presents an axiomatic model of probabilistic binary choice (incremental expected utility advantage model) that does not allow for any violations of stochastic dominance. A popular random utility model (e.g. Loomes and Sugden, 1995; Gul and Pesendorfer, 2006) also rules out the violations of stochastic dominance. 
This paper presents a new axiomatic model of probabilistic choice that can be summarized as follows. Let $X=\left\{x_{1}, \ldots, x_{n}\right\}$ be a set of outcomes (consequences) numbered so that an individual always chooses outcome $x_{i}$ over outcome $x_{j}$ when $i>j$. Let $\mathscr{L}$ be a set of probability distributions (lotteries) on $X$. Lottery $A\left(a_{1}, \ldots, a_{n}\right) \in \mathscr{L}$ weakly dominates lottery $B\left(b_{1}, \ldots, b_{n}\right) \in \mathscr{L}$, denoted as $A \gtrsim B$, if $\sum_{i=1}^{j} a_{i} \leq \sum_{i=1}^{j} b_{i}$ for any $j \in\{1, \ldots, n\}$. The partially ordered set $(\mathscr{L}, \underset{\sim}{\sim})$ is a lattice i.e. for any two lotteries $A, B \in \mathscr{L}$ there exists a least upper bound $A \vee B$ and a greatest lower bound $A \wedge B$. According to the proposed model of probabilistic choice, an individual chooses lottery $A$ over another lottery $B$ with probability

$$
P(A, B)=\frac{\varphi(U(A)-U(A \wedge B))}{\varphi(U(A)-U(A \wedge B))+\varphi(U(B)-U(A \wedge B))},
$$

where $U: \mathscr{L} \rightarrow \mathbb{R}$ is the expected utility function and $\varphi: \mathbb{R}_{+} \rightarrow \mathbb{R}_{+}$is a non-decreasing function with $\varphi(0)=0$.

Model (1) has several intuitive properties. An individual always chooses $A$ over $B$ if $A$ stochastically dominates $B$ (in this case $B=A \wedge B$ ). Similarly, an individual never chooses $A$ over $B$ if $B$ stochastically dominates $A$ (in this case $A=A \wedge B$ ). An individual chooses $A$ over $B$ with probability $P(A, B) \geq 0.5$ if $U(A) \geq U(B)$. Classical expected utility theory is a limiting case of (1) when $\varphi(z)=z^{\lambda}$ and $\lambda \rightarrow+\infty$. When lotteries have no more than three outcomes, model (1) coincides with a random utility model if function $\varphi($.$) is$ homogeneous.

Binary choice probabilities admit representation (1) if and only if they satisfy the following axioms: completeness, weak stochastic transitivity, continuity, common 
consequence independence, outcome monotonicity and odds ratio independence. The proposed model of probabilistic choice is compatible with several well-known violations of expected utility theory such as the common ratio effect (e.g. Allais, 1953) and the violations of the betweenness property (e.g. Camerer and Ho, 1994).

The proposed model of probabilistic choice has a simple interpretation. Stochastic dominance imposes a greatest lower bound on every pair of lotteries. This greatest lower bound serves as an endogenous reference lottery. An individual compares two lotteries relative to this natural referent. The closer is lottery $A(B)$ to the reference lottery $A \wedge B$ in terms of expected utility, the closer is the choice probability $P(A, B)$ to zero (one).

Model (1) has the same functional form as the strict utility model discussed in Becker et al. (1963) with the only difference that lotteries are compared to a reference lottery $A \wedge B$. In other words, we can consider a strict utility model as a variation of choice rule (1) when the reference lottery is zero for all lottery pairs. In contrast, the model of probabilistic choice proposed in this paper has an endogenous reference lottery $A \wedge B$. This reference lottery captures the context of a decision problem. In particular, it allows a decision maker to avoid violations of stochastic dominance.

The remainder of this paper is organized as follows. Section II presents six axioms imposed on a primitive binary choice probability function (a fuzzy preference relation) and discusses their descriptive validity. Section III contains the main result of the paper-a representation theorem for binary choice probabilities and discusses its main implications and possible extensions. Section IV generalizes the proposed model from binary choice to choice among $m>2$ alternatives. Section V concludes. 


\section{Axioms}

Let $X=\left\{x_{1}, \ldots, x_{n}\right\}$ denote a finite set of outcomes (consequences). These outcomes can be monetary payoffs, consumption bundles, portfolios of assets, health states etc. Objects of choice are risky lotteries. A risky lottery $A\left(a_{1}, \ldots, a_{n}\right)$ is a probability distribution on $X$ i.e. it delivers outcome $x_{i}$ with a probability $a_{i} \in[0,1], i \in\{1, \ldots, n\}$, and $\sum_{i=1}^{n} a_{i}=1$. A degenerate lottery that yields one outcome $x_{i} \in X$ with probability one is denoted by $X_{i}$. A compound lottery $\alpha A+(1-\alpha) B$ yields a risky lottery $A$ with probability $\alpha \in[0,1]$ and a risky lottery $B$ with probability $1-\alpha$. The set of all risky lotteries is denoted by $\mathscr{L}$.

The primitive of choice is a binary choice probability function $P: \mathscr{L} \times \mathscr{L} \rightarrow[0,1]$, which is also known as a fuzzy preference relation (e.g. Zimmerman et al., 1984). $P(A, B)$ represents the probability that an individual chooses lottery $A$ over lottery $B$ in a direct binary choice. For any two lotteries $A, B \in \mathscr{L}, A \neq B$, probability $P(A, B)$ is observable from a relative frequency with which an individual chooses $A$ when he is asked to choose repeatedly between $A$ and $B$. Probability $P(A, A), A \in \mathscr{L}$, cannot be observed from actual choices and it is not defined. We now present six axioms imposed on binary choice probability function.

Axiom 1 (Completeness) $P(A, B)+P(B, A)=1$ for any lotteries $A, B \in \mathscr{L}, A \neq B$.

Axiom 1 states that only two events are possible in a binary choice between $A$ and $B$ - either an individual chooses $A$ over $B$ or an individual chooses $B$ over $A$.

Axiom 2 (Weak Stochastic Transitivity) For any three lotteries $A, B, C \in \mathscr{L}$ such that $A \neq B \neq C$ the following holds:

a) if $P(A, B) \geq 0.5$ and $P(B, C) \geq 0.5$ then $P(A, C) \geq 0.5$;

b) if $P(A, B)=1$ and $P(B, C)=1$ then $P(A, C)=1$. 
Axiom 2a imposes a basic consistency requirement on binary choice probabilities. If an individual is likely to choose $A$ over $B$ and he is also likely to choose $B$ over $C$, then this individual is also likely to choose $A$ over $C$. Empirical data generally support weak stochastic transitivity (e.g. Luce and Suppes, 1965; Tversky and Russo, 1969; Luce, 1977). Part b) of Axiom 2 slightly strengthens traditional weak stochastic transitivity. In particular, it postulates that if an individual always chooses $A$ over $B$ and he always chooses $B$ over $C$, then this individual always chooses $A$ over $C$.

Axiom 3 (Continuity) The sets $\{\alpha \in[0,1] \mid P(A, \alpha B+(1-\alpha) C) \geq 0.5\}$ and $\{\alpha \in[0,1] \mid P(A, \alpha B+(1-\alpha) C) \leq 0.5\}$ are closed for any three lotteries $A, B, C \in \mathscr{L}$, such that $A \neq \alpha B+(1-\alpha) C$ for some $\alpha \in[0,1]$.

Axiom 3 states that if an individual is likely (unlikely) to choose a lottery over each element of a converging sequence of lotteries then he is also likely (unlikely) to choose this lottery over the limit of the sequence. Continuity rules out the possibility of lexicographic choices (e.g. an individual cares first of all for the prospect of getting at least something for sure). Continuity is a necessary condition for many popular models of probabilistic choice such as the Fechner model of random errors, the Luce choice model and the constant error/tremble model (e.g. Blavatskyy, 2008).

Axioms 1-3 are straightforward translations of the axioms of expected utility theory, when a primitive binary choice probability function is used instead of a primitive binary preference relation.

Axiom 4 (Common Consequence Independence) $P(\alpha A+(1-\alpha) C, \alpha B+(1-\alpha) C)=$ $=P(\alpha A+(1-\alpha) D, \alpha B+(1-\alpha) D)$ for any lotteries $A, B, C, D \in \mathscr{L}, A \neq B$, and any $\alpha \in(0,1]$. 
Axiom 4 states that binary choice probabilities are independent of the consequences that are common to both choice alternatives. In other words, if two lotteries yield identical chances of the same outcome (or, more generally, if two compound lotteries yield identical chances of the same risky lottery) this common consequence does not affect the probability of one alternative being chosen over the other.

Axiom 4 is weaker than the stochastic analogue of the independence axiom of expected utility theory, which requires that $P(A, B)=P(\alpha A+(1-\alpha) C, \alpha B+(1-\alpha) C)$ for any $A, B, C \in \mathscr{L}, A \neq B$, and any $\alpha \in(0,1]$. Thus, common consequence independence is compatible with several well-known empirical violations of expected utility theory. For example, Axiom 4 allows certain types of the common ratio effect (Loomes, 2005) as well as the violations of the betweenness property (Blavatskyy, 2006a). Blavatskyy (2008) shows that common consequence independence is a necessary axiom for the Fechner model of random errors, the Luce choice model and the constant error/tremble model.

Proposition 1 If Axioms 1, 2a, 3 and 4 hold then there exists an assignment of real numbers $u_{i}$ to every outcome $x_{i}, i \in\{1, \ldots, n\}$, such that $P(A, B)=0.5$ if and only if $\sum_{i=1}^{n} u_{i} a_{i}=\sum_{i=1}^{n} u_{i} b_{i}$ for any two lotteries $A\left(a_{1}, \ldots, a_{n}\right), B\left(b_{1}, \ldots, b_{n}\right) \in \mathscr{L}, A \neq B$

Proof is presented in the Appendix.

The assignment of real numbers $\left(u_{1}, \ldots, u_{n}\right)$ is unique up to a linear transformation. If a vector $\mathbf{u}=\left(u_{1}, \ldots, u_{n}\right)$ represents binary choice probabilities of 0.5 then another vector $k \mathbf{u}+m, k \neq 0$, does as well. The vector of real numbers $\left(u_{1}, \ldots, u_{n}\right)$ can be interpreted as a vector of von Neumann-Morgenstern utilities (von Neumann and Morgenstern, 1944). 
Axiom 5 (Outcome Monotonicity) $P\left(X_{i}, \alpha X_{i}+(1-\alpha) X_{j}\right) \in\{0,1\}$ for any $i, j \in\{1, \ldots, n\}, i \neq j$ and any $\alpha \in[0,1)$.

If we set $\alpha=0$ then Axiom 5 states that choice under certainty is deterministic. If an individual faces a choice between two sure things, then he always chooses the same alternative. This is particularly appealing when lottery outcomes are monetary payoffs and people care for money. For instance, it appears quite intuitive that a sure prospect of receiving $£ 30$ is always chosen over a sure prospect of receiving $£ 10 .{ }^{1}$

More generally, Axiom 5 states that choice between a sure outcome and a binary lottery that involves this outcome is deterministic. Again this is quite intuitive if lottery outcomes can be ordered in a natural way. For instance, a prospect of a healthy life is always preferred over a medical treatment that provides full recovery with probability $\alpha<1$. Axiom 5 is a weaker version of the principle of internality, which is used in several models of probabilistic choice (e.g. Blavatskyy 2006b; 2007) and appears to be descriptively valid. ${ }^{2}$

If Axioms 1, 2b and 5 hold, then, without loss of generality, we can number lottery outcomes so that $P\left(X_{i}, X_{j}\right)=1$ if $i>j$, for any $i, j \in\{1, \ldots, n\}$.

Definition 1 Lottery $A\left(a_{1}, \ldots, a_{n}\right) \in \mathscr{L}$ weakly dominates lottery $B\left(b_{1}, \ldots, b_{n}\right) \in \mathscr{L}$, denoted as $A \stackrel{d}{\gtrsim}$, if $\sum_{i=1}^{j} a_{i} \leq \sum_{i=1}^{j} b_{i}$ for any $j \in\{1, \ldots, n\}$.

Our definition of weak dominance is closely related to the concept of stochastic dominance for lotteries over monetary outcomes. If lottery outcomes are monetary payoffs, then lottery $A$ first-order stochastically dominates lottery $B$ if and only if $A \stackrel{d}{\approx}$ and $A \neq B$.

\footnotetext{
${ }^{1}$ In fact, it is so intuitive that I am not aware of a single experimental study that attempts to test this. ${ }^{2}$ I know only one study, Gneezy et al. (2006), that reports violations of internality in indirect choice and pricing tasks. Recently, Ortmann et al. (2007) argued that the results of Gneezy et al. (2006) may be an artifact of a between-subject experimental design. In a private correspondence, Gneezy et al. (2006) informed me that they did not find violations of internality in a direct binary choice.
} 
Proposition 2 If Axioms 1, 2b, 3-5 hold then $P(A, B)=1$ for any two lotteries $A, B \in \mathscr{L}$

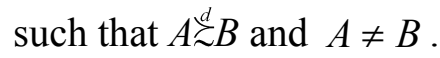

Proof is presented in the Appendix.

If lottery outcomes are monetary payoffs, Proposition 2 states that people never violate first-order stochastic dominance. Experimental evidence shows that people seldom violate transparent stochastic dominance. For instance, Carbone and Hey (1995) find only one violation of transparent dominance in 320 decisions (rate of violation $0.3 \%$ ). Loomes and Sugden (1998) discover 12 such violations in 920 choice decisions (rate of violation 1.3\%). Hey (2001) reports 24 violations of transparent dominance in 1590 choice decisions (rate of violation 1.5\%). Loomes et al. (2002) argue that such rare instances can result from a lapse of concentration and they can be captured by adding a tremble term to the core model of probabilistic choice.

Experimental evidence shows that people can violate stochastic dominance much more frequently if two lotteries are framed so that the dominance relation is not transparent (e.g. Tversky and Kahneman, 1986; Birnbaum, 2004; 2005a; 2005b). However, such violations appear to result from improper understanding of a decision problem. When the same binary choice problem is presented in a different format so that the dominance relation becomes transparent, the rates of violation significantly decrease (e.g. Tversky and Kahneman, 1986; Birnbaum, 2004). Hence, the implications of Proposition 2 appear to be descriptively acceptable provided that people comprehend stochastic dominance when they face it.

Proposition 3 The partially ordered set $(\mathscr{L}, \stackrel{d}{\sim})$ is a lattice.

Proof is presented in the Appendix. 
Proposition 3 states that for any two lotteries $A, B \in \mathscr{L}$ there exists a least upper bound, denoted by $A \vee B$, and a greatest lower bound, denoted by $A \wedge B$. One can argue that lotteries $A \vee B$ and $A \wedge B$ form a natural context for a binary choice problem because they are the best and the worst prospect that an individual can possibly obtain from a given decision problem. ${ }^{3}$ Marley (1997) discusses the role of such natural contextual reference points in different models of probabilistic choice.

Axiom 6 (Odds Ratio Independence) For any three lotteries $A, B, C \in \mathscr{L}$ such that $A \neq B, A \neq C$ and $A \wedge B=A \wedge C$ the odds ratio $\frac{P(A, B)}{P(B, A)} / \frac{P(A, C)}{P(C, A)}$ is independent of $A$.

Axiom 6 can be illustrated with the following example. Consider an individual who chooses where to go for lunch. There are three restaurants close to his office. An Egyptian bistro is located around the corner. There are also Thai and Swiss restaurants, both of which are two blocks away. If we compare the relative chance that the Egyptian restaurant is chosen over the Thai restaurant with the relative chance that the Egyptian restaurant is chosen over the Swiss restaurant, this odds ratio should depend only on the characteristics of Thai and Swiss restaurants and it should not depend on the characteristics of the Egyptian bistro.

Intuitively, Axiom 6 plays the role of an exchange rate. Axiom 6 allows us to bring various choice probabilities to a common denominator. Such an axiom is not required in traditional deterministic decision theories because they deal with degenerate choice probabilities that take only two values - either one or zero. Since Axiom 6 is a new axiom that is not used in the existing axiomatic models of probabilistic choice, I am not aware of any empirical tests of Axiom 6.

\footnotetext{
${ }^{3}$ This interpretation was suggested to me by Tony Marley.
} 


\section{Representation Theorem}

Proposition 4 The binary choice probability function $P: \mathscr{L} \times \mathscr{L} \rightarrow[0,1]$ satisfies Axioms 1-6 if and only if there exists an assignment of real numbers $u_{i}$ to every outcome $x_{i}, i \in\{1, \ldots, n\}$, and there exists a non-decreasing function $\varphi: \mathbb{R}_{+} \rightarrow \mathbb{R}_{+}$with $\varphi(0)=0$, such that for any two lotteries $A\left(a_{1}, \ldots, a_{n}\right), B\left(b_{1}, \ldots, b_{n}\right) \in \mathscr{L}, A \neq B$, the probability $P(A, B)$ can be written as

$$
P(A, B)=\frac{\varphi\left(\sum_{i=1}^{n} u_{i} a_{i}-\sum_{i=1}^{n} u_{i} c_{i}\right)}{\varphi\left(\sum_{i=1}^{n} u_{i} a_{i}-\sum_{i=1}^{n} u_{i} c_{i}\right)+\varphi\left(\sum_{i=1}^{n} u_{i} b_{i}-\sum_{i=1}^{n} u_{i} c_{i}\right)},
$$

where $c_{i}=\max \left\{\sum_{j=1}^{i} a_{j}, \sum_{j=1}^{i} b_{j}\right\}-\max \left\{\sum_{j=1}^{i-1} a_{j}, \sum_{j=1}^{i-1} b_{j}\right\}$.

Proof is presented in the Appendix.

When lottery outcomes are monetary payoffs, formula (2) implies that $P(A, B)=1$ if $A$ stochastically dominates $B,{ }^{4}$ and that $P(A, B)=0$ if $B$ stochastically dominates $A .{ }^{5}$ Formula (2) also implies that $P(A, B)=0.5$ if the expected utility of lottery $A$ is exactly equal to the expected utility of lottery $B$.

Note that lottery $A \wedge B$ yields outcome $x_{i}$ with probability $c_{i}, i \in\{1, \ldots, n\} .{ }^{6}$ Thus, one possible interpretation of model (2) is the following. Lotteries $A$ and $B$ are evaluated relative to the endogenous reference lottery, which is their greatest lower bound $A \wedge B$. The closer is lottery $A(B)$ to the reference lottery $A \wedge B$ in terms of expected utility, the closer is binary choice probability $P(A, B)$ to zero (one). Function $\varphi($.$) captures the$ sensitivity of binary choice probabilities to changes in the expected utility of lotteries relative to the endogenous reference lottery.

\footnotetext{
${ }^{4}$ In this case $c_{i}=b_{i}$ for all $i \in\{1, \ldots, n\}$.

${ }^{5}$ In this case $c_{i}=a_{i}$ for all $i \in\{1, \ldots, n\}$.

${ }^{6}$ See proof of Proposition 3.
} 
Models of probabilistic choice can be conveniently presented in the probability triangle (e.g. Machina, 1982). When lotteries have no more than three outcomes $(n=3)$, the set of all lotteries $\mathscr{L}$ can be represented as a rectangular triangle. The probability of the first outcome $\left(b_{1}\right)$ is conventionally shown on the horizontal axis. The probability of the third outcome $\left(b_{3}\right)$ is typically shown on the vertical axis. We will select an arbitrary lottery $A$ inside the probability triangle and then plot the set of all other lotteries $B$ such that $P(A, B)$ is constant.

Figure 1 shows "probabilistic indifference sets" $\{B \in \mathscr{L} \mid P(A, B)=r\}$ for a selected lottery $A \in \mathscr{L}$ and various choice probabilities $r \in[0,1]$. Figure 1a) provides an illustration for a constant error/tremble model. In a constant error/tremble model, the probability $r$ can take only three possible values. Figure 1b) illustrates the Fechner model of random errors and the Luce choice model. ${ }^{7}$ In these two models the sets $\{B \in \mathscr{L} \mid P(A, B)=r\}$ are parallel straight lines with a positive slope. Figure 1c) illustrates a random utility model. The random utility model is a special case of equation (2) if the function $\varphi($.$) is homogeneous and lotteries$ have up to three outcomes. ${ }^{8}$

Figure 1d) illustrates the model of probabilistic choice described in equation (2) when the function $\varphi($.$) is not homogeneous. Note that in model (2) the set \{B \in \mathscr{L} \mid P(A, B)=r\}$ is a straight line with a positive slope only if $r=0.5$. The set $\{B \in \mathscr{L} \mid P(A, B)=1\}$ contains all lotteries that are stochastically dominated by $A$. Similarly, the set $\{B \in \mathscr{L} \mid P(A, B)=0\}$ contains all lotteries that stochastically dominate lottery $A$.

\footnotetext{
${ }^{7}$ We can obtain the Luce choice model from formula (2) if we assume that function $\varphi($.$) is the exponential$ function. However, the Luce choice model is not a special case of our proposed model of probabilistic choice because the exponential function does not satisfy the boundary condition $\varphi(0)=0$.

${ }^{8}$ When lotteries have more than three outcomes neither the random utility model nor the model presented in equation (2) are special cases of each other.
} 


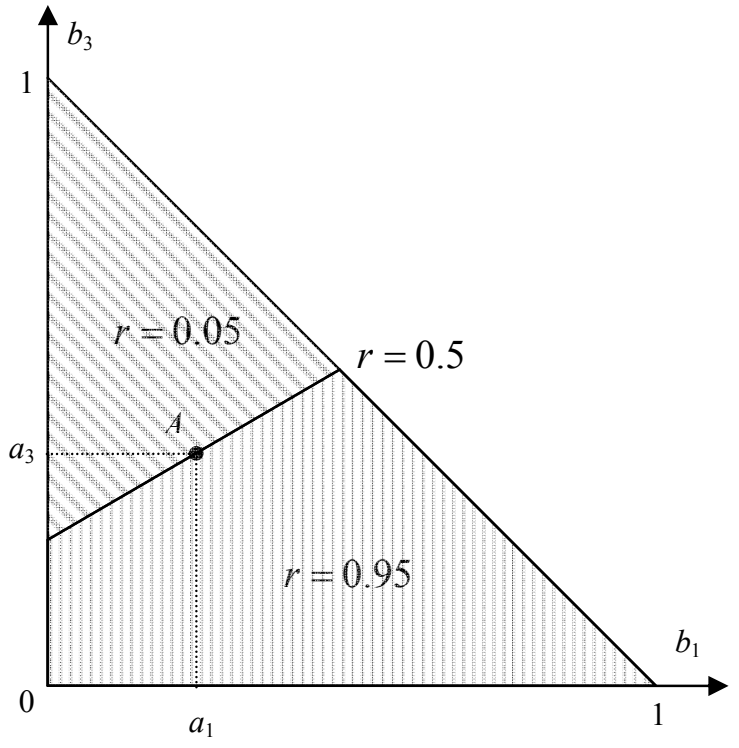

a) Constant error/tremble model

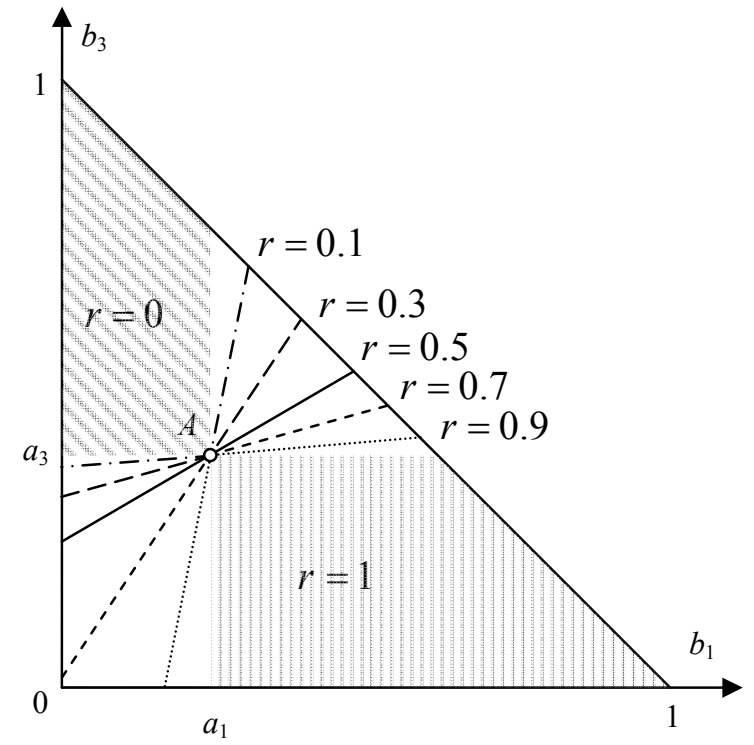

c) Random utility/Model (2), $\varphi($.$) is homogeneous$

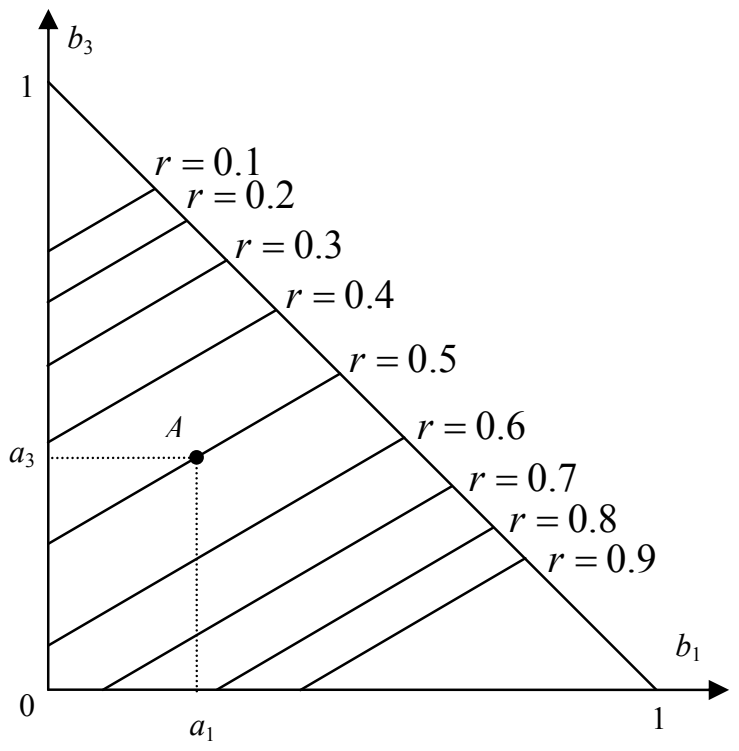

b) Fechner model/Luce choice model

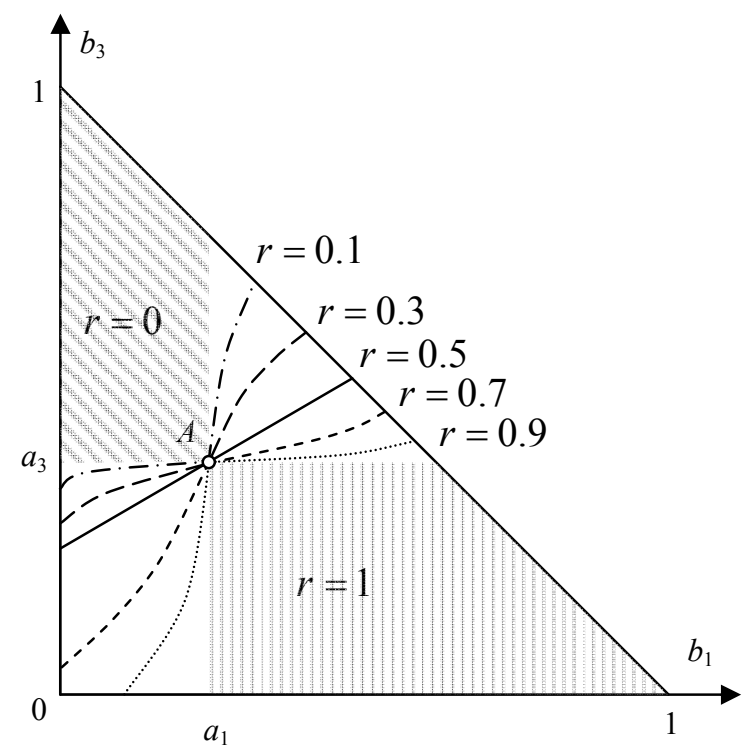

d) Model (2), $\varphi($.$) is not homogeneous$

Figure 1 Models of probabilistic choice presented in the probability triangle.

The set $\mathscr{L}$ of all lotteries that yield no more than three outcomes is represented by a rectangular triangle. The probability of the first outcome $\left(b_{1}\right)$ is shown on the horizontal axis. The probability of the third outcome $\left(b_{3}\right)$ is shown on the vertical axis. Each panel shows the sets $\{B \in \mathscr{L} \mid P(A, B)=r\}$ for one lottery $A \in \mathscr{L}$ and various choice probabilities $r \in[0,1]$. 
Nonlinearity of choice probabilities illustrated by Figure 1d) suggests that model (2) can explain some violations of expected utility theory if the function $\varphi($.$) is not$ homogeneous. For instance, model (2) is compatible with certain types of the common ratio effect as well as with violations of the betweenness property.

The common ratio effect is typically illustrated with lotteries over three monetary outcomes $x_{3}>x_{2}>x_{1}=0$. The effect is observed when $P(A, B)>P(C, D)$ for lotteries $A(0,1,0)$, $B(1-q, 0, q), C(1-\theta, \theta, 0), D(1-\theta q, 0, \theta q)$, and probabilities $q, \theta \in(0,1)$ (e.g. Allais, 1953). If choice probabilities admit representation (2) then $P(A, B)>P(C, D)$ if the following holds:

$$
\frac{\varphi\left((1-q)\left(u_{2}-u_{1}\right)\right)}{\varphi\left(q\left(u_{3}-u_{2}\right)\right)}>\frac{\varphi\left(\theta(1-q)\left(u_{2}-u_{1}\right)\right)}{\varphi\left(\theta q\left(u_{3}-u_{2}\right)\right)} .
$$

If function $\varphi($.$) is homogeneous then the left hand side and the right hand side of (3) are$ exactly equal. In this case model (2) coincides with a random utility model, which cannot explain the common ratio effect. However, if function $\varphi($.$) is not homogeneous then$ inequality (3) may easily hold.

The violations of the betweenness property are usually illustrated with two lotteries $A$ and $B, A \neq B$, and a compound lottery $M=\alpha A+(1-\alpha) B, \alpha \in(0,1)$. An individual is said to reveal quasi-concave preferences if he chooses $A$ over $B$ and $M$ over $A$. An individual is said to reveal quasi-convex preferences if he chooses $B$ over $A$ and $A$ over $M$. Systematic violations of the betweenness are observed when people reveal quasi-concave preferences more often than quasi-convex preferences or vice versa (e.g. Camerer and Ho, 1994). If binary choices are independent then the violations of the betweenness are observed if $P(A, B) \neq P(A, M)$. If choice probabilities admit representation (2) then $P(A, B) \neq P(A, M)$ if and only if the following condition holds: 


$$
\frac{\varphi(U(A)-U(A \wedge B))}{\varphi(U(B)-U(A \wedge B))} \neq \frac{\varphi((1-\alpha) \cdot[U(A)-U(A \wedge B)])}{\varphi((1-\alpha) \cdot[U(B)-U(A \wedge B)])}
$$

where $U($.$) denotes the expected utility of a corresponding lottery. If the function \varphi($.$) is not$ homogeneous then condition (4) holds for at least one pair of lotteries $A$ and $B$. Thus, model (2) is compatible with the violations of the betweenness property.

It is interesting to note that for any two lotteries $A, B \in \mathscr{L}$ a compound lottery $0.5 A+0.5 B$ is equivalent to a compound lottery $0.5(A \vee B)+0.5(A \wedge B)$. This implies that $U(A)+U(B)=U(A \vee B)+U(A \wedge B)$ and we can rewrite equation (2) as follows:

$$
\begin{aligned}
P(A, B) & =\frac{\varphi(U(A)-U(A \wedge B))}{\varphi(U(A)-U(A \wedge B))+\varphi(U(B)-U(A \wedge B))}= \\
& =\frac{\varphi(U(A \vee B)-U(B))}{\varphi(U(A \vee B)-U(A))+\varphi(U(A \vee B)-U(B))}
\end{aligned}
$$

Thus, in this model, we can compare lotteries relative to their greatest lower bound, or, equivalently, relative to their least upper bound. The closer is lottery $A(B)$ to lottery $A \vee B$ in terms of expected utility, the closer is binary choice probability $P(A, B)$ to one (zero).

A natural extension of this model is to weaken the axiom of common consequence independence to accommodate, for example, choice patterns observed in the Allais paradox (Allais, 1953). Axiom 4 can be replaced by the axioms of non-expected utility theories, provided that these axioms are reformulated in terms of a primitive binary choice probability function rather than a primitive binary preference relation. However, such extensions may require different binary operations $\vee$ and $\wedge$ on $\mathscr{L}$ than those derived from

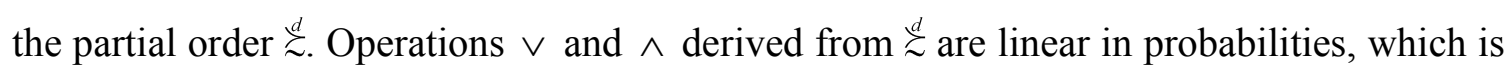
a convenient property in conjunction with Axiom 4, but it may be unnecessary if Axiom 4 is relaxed. 


\section{Choice among $m>2$ alternatives}

A model of binary choice can be generalized to choice among $m>2$ alternatives. Luce and Suppes (1965, pp. 351-352) give an example of the following algorithm. When choosing among $m>2$ alternatives, an individual first selects two alternatives at random and chooses between them. The chosen alternative is then compared to another randomly selected alternative. This step is subsequently repeated i.e. the individual chooses between a previously chosen alternative and another randomly selected element of the choice set $S=\left\{A_{1}, \ldots, A_{m}\right\}$. Let $Q\left(A_{i} \mid S\right)_{t}$ denote the probability that the $i$-th alternative $A_{i}, i \in\{1, \ldots, m\}$, is chosen over another randomly selected alternative at the iteration $t \in \mathbb{N}$ of this algorithm. Probabilities $Q\left(A_{i} \mid S\right)_{t}$ are recursively defined by the following system of equations:

(6)

$$
\left(\begin{array}{c}
Q\left(A_{1} \mid S\right)_{t+1} \\
Q\left(A_{2} \mid S\right)_{t+1} \\
\ldots \\
Q\left(A_{m} \mid S\right)_{t+1}
\end{array}\right)=\frac{1}{m-1}\left(\begin{array}{cccc}
\sum_{i=1}^{m} P\left(A_{1}, A_{i}\right) & P\left(A_{1}, A_{2}\right) & \ldots & P\left(A_{1}, A_{m}\right) \\
P\left(A_{2}, A_{1}\right) & \sum_{\substack{i=1 \\
i \neq 2}}^{m} P\left(A_{2}, A_{i}\right) & \ldots & P\left(A_{2}, A_{m}\right) \\
\ldots & \ldots & \ddots & \ldots \\
P\left(A_{m}, A_{1}\right) & P\left(A_{m}, A_{2}\right) & \ldots & \sum_{\substack{i=1 \\
i \neq m}}^{m} P\left(A_{m}, A_{i}\right)
\end{array}\right) \cdot\left(\begin{array}{c}
Q\left(A_{1} \mid S\right)_{t} \\
Q\left(A_{2} \mid S\right)_{t} \\
\ldots \\
Q\left(A_{m} \mid S\right)_{t}
\end{array}\right)
$$

with the initial condition $Q\left(A_{i} \mid S\right)_{1}=1 / m$ for all $i \in\{1, \ldots, m\}$.

The probability that $A_{i}$ is chosen from the set $S$ is defined as $Q\left(A_{i} \mid S\right) \equiv \lim _{t \rightarrow \infty} Q\left(A_{i} \mid S\right)_{t}$ for all $i \in\{1, \ldots, m\}$. In other words, we calculate the chance that an individual chooses the $i$ th alternative from the set $S$ as if the individual continues the sequence of binary choices described above ad infinitum. A vector $\mathbf{Q}=\left(Q\left(A_{1} \mid S\right), \ldots, Q\left(A_{m} \mid S\right)\right)^{\prime}$ is an asymptotic probability distribution on $S$, which solves the following homogeneous matrix equation

$$
(\mathbf{P}-(m-1) \mathbf{I}) \cdot \mathbf{Q}=0,
$$

where $\mathbf{P}$ is a transition matrix on the right-hand side of (6) and $\mathbf{I}$ is the $m \times m$ identity matrix. 
Before presenting a solution to (7), it is convenient to introduce the following notation. Let $G$ denote an arborescence with the vertex set $S$ and let $\Gamma(\mathrm{S})$ be the set of all arborescences with the vertex set $S$. Let $R(G)$ be the root of $G$ and let $E(G)$ be the edge set of $G$. Note that the elements of $E(G)$ are ordered pairs of lotteries $\{A, B\}$ such that $A, B \in S$ and $A \neq B$. The product $\prod_{\{A, B\} \in E(G)} P(A, B)$ is the probability that an individual chooses the initial vertex (tail) $A$ over the terminal vertex (head) $B$ in all edges of an arborescence $G$. With this notation, vector $\mathbf{Q}$ that solves (7) can be written as

(8) $\mathbf{Q}=\lambda\left(\sum_{G \in \Gamma(S) \mid R(G)=A_{1}} \prod_{\{A, B\} \in E(G)} P(A, B), \ldots, \sum_{G \in \Gamma(S) \mid R(G)=A_{m}} \prod_{\{A, B\} \in E(G)} P(A, B)\right)^{\prime}$, where $\lambda$ is an arbitrary constant.

Since choice probabilities should add up to one, i.e. $\sum_{i=1}^{m} Q\left(A_{i} \mid S\right)=1$, it follows immediately that $\lambda=1 / \sum_{G \in \Gamma(S)} \prod_{\{A, B\} \in E(G)} P(A, B)$. Thus, an individual chooses a lottery $A_{i} \in S$ from a non-singleton choice set $S$ with a probability

$$
Q\left(A_{i} \mid S\right)=\frac{\sum_{G \in \Gamma(S) \mid R(G)=A_{i}} \prod_{\{A, B\} \in E(G)} P(A, B)}{\sum_{G \in \Gamma(S)} \prod_{\{A, B\} \in E(G)} P(A, B)},
$$

where binary choice probabilities $P(A, B)$ are given in equation (5) and $i \in\{1, \ldots, m\}$.

Equation (9) allows us to generalize any model of binary choice to choice among $m>2$ alternatives and it has several intuitive properties. For example, if an individual always (never) chooses one lottery over every other element of a choice set in a direct binary choice, then he also always (never) chooses this lottery from the overall choice set. If an individual chooses with probabilities $50 \%-50 \%$ between any two lotteries from a given choice set, then each lottery is selected with equal probability $(1 / m)$ from this choice set. 


\section{Conclusion}

This paper presents a simple axiomatic model of decision making under risk. In this model, when it comes to choosing one lottery over another, each alternative has a chance of being selected, unless one lottery stochastically dominates the other. It is straightforward to apply the proposed model in empirical research. In order to estimate a binary choice probability a researcher needs to calculate only the expected utilities of three lotteries - the two lotteries that are compared and their greatest lower bound (or their least upper bound) in terms of weak dominance. The greatest lower bound or the least upper bound serves as a natural endogenous reference lottery to which lotteries are compared.

The representation theorem shows that the necessary and sufficient conditions for the model are four relatively standard axioms (completeness, weak stochastic transitivity, continuity and common consequence independence) and two relatively new axioms (outcome monotonicity and odds ratio independence). Outcome monotonicity requires that choice under certainty is deterministic. Odds ratio independence connects binary choice probabilities of different values. Both standard and new axioms are formulated in terms of a primitive binary choice probability function. Thus, they can be easily tested on experimental data.

The proposed model is compatible with several well-known violations of expected utility theory. In particular, the model can accommodate the common ratio effect and violations of the betweenness. The proposed model is also quite general. It can be applied to a variety of choice situations, where lottery outcomes are not necessarily monetary payoffs. Finally, although the baseline model is developed for binary choice, it can be easily extended to choice situations where a decision maker faces $m>2$ alternatives. 


\section{References}

Aczél, J. (1966) "Lectures on functional equations and their applications" London: Academic Press

Allais, M. (1953) “Le comportement de l'homme rationnel devant le risque: critique des postulates et axiomes de l'école Américaine" Econometrica 21, 503-546

Ballinger, P. and N. Wilcox (1997) "Decisions, error and heterogeneity" Economic Journal 107, 1090-1105

Becker, Gordon M., Morris H. DeGroot, and Jacob Marschak (1963) "Stochastic Models of Choice Behavior" Behavioral Science 8, 41-55

Birnbaum, M. (2004) "Tests of rank-dependent utility and cumulative prospect theory in gambles represented by natural frequencies: effects of format, event framing, and branch splitting" Organizational Behavior and Human Decision Processes 95, 40-65

Birnbaum, M. (2005a) "Three new tests of independence that differentiate models of risky decision making" Management Science 51, 1346-1358

Birnbaum, M. (2005b) "A comparison of five models that predict violations of firstorder stochastic dominance in risky decision making" Journal of Risk and Uncertainty 31, 263-287

Blavatskyy, Pavlo (2008) "Stochastic Utility Theorem" Journal of Mathematical Economics, forthcoming

Blavatskyy, Pavlo (2007) "Stochastic Expected Utility Theory” Journal of Risk and Uncertainty 34, 259-286

Blavatskyy, Pavlo (2006a) "Violations of Betweenness or Random Errors?" Economics Letters 91(1), 34-38

Blavatskyy, Pavlo (2006b) "Stochastic Choice under Risk", IEW Working Paper \#272, available at http://www.iew.unizh.ch/wp/iewwp272.pdf

Buschena, D. and Zilberman, D. (2000) "Generalized expected utility, heteroscedastic error, and path dependence in risky choice" Journal of Risk and Uncertainty 20, 67-88

Camerer, C. (1989) "An experimental test of several generalized utility theories." Journal of Risk and Uncertainty 2, 61-104 
Camerer, C. and Ho, T. (1994) "Violations of the Betweenness Axiom and Nonlinearity in Probability" Journal of Risk and Uncertainty 8, 167-196

Carbone, E. (1997) "Investigation of stochastic preference theory using experimental data" Economics Letters 57, 305-311

Carbone, E., and Hey, J. (1995) "A comparison of the estimates of EU and non-EU preference functionals using data from pairwise choice and complete ranking experiments" Geneva Papers on Risk and Insurance Theory 20, 111-133

Gneezy, Uri, John List, and George Wu. (2006) "The Uncertainty Effect: When a Risky Prospect is Valued Less than its Worst Possible Outcome," Quarterly Journal of Economics 121, 1283-1309

Gul, F. and Pesendorfer, W. (2006) "Random Expected Utility" Econometrica 71(1), 121-146

Fechner, G. (1860) "Elements of Psychophysics" NY: Holt, Rinehart and Winston

Fishburn, P. (1978) A probabilistic expected utility theory of risky binary choices, International Economic Review 19, 633-646

Harless, D. and C. Camerer (1994) The predictive utility of generalized expected utility theories, Econometrica 62, 1251-1289

Hey, J. (1995) "Experimental investigations of errors in decision making under risk" European Economic Review 39, 633-640

Hey, J. (2001) “Does repetition improve consistency?” Experimental Economics 4, 5-54

Hey, J.D. and C. Orme (1994) Investigating generalisations of expected utility theory using experimental data, Econometrica 62, 1291-1326

Loomes, G. (2005) "Modelling the stochastic component of behaviour in experiments: some issues for the interpretation of data" Experimental Economics 8 301-323

Loomes, G. and Sugden, R. (1995) "Incorporating a stochastic element into decision theories" European Economic Review 39, 641-648

Loomes, G. and Sugden, R. (1998) "Testing different stochastic specifications of risky choice" Economica $\mathbf{6 5}, 581-598$

Loomes, G., Moffatt, P. and Sugden, R. (2002) "A microeconomic test of alternative stochastic theories of risky choice" Journal of Risk and Uncertainty 24, 103-130 
Luce, R.D. (1977) "The Choice Axiom after Twenty Years" Journal of Mathematical Psychology 15, 215-233

Luce, R. D. and Suppes, P. (1965) "Preference, utility, and subjective probability" Handbook of mathematical psychology, Vol. III, 249-410, Wiley, New York NY

Machina, M. (1982) “Expected utility' analysis without the independence axiom", Econometrica 50, 277-323

Marley, A. A. J. (1997) "Probabilistic choice as a consequence of nonlinear (sub) optimization" Journal of Mathematical Psychology 41, 382-391

Ortmann, Andreas, Sasha Prokosheva, Ondrej Rydval and Ralph Hertwig (2007) "Valuing A Risky Prospect Less Than Its Worst Outcome: Uncertainty Effect or Task Ambiguity?" CERGE-EI working paper 334

Schmidt, U. and Hey, J. (2004) “Are Preference Reversals Errors? An Experimental Investigation" Journal of Risk and Uncertainty 29, 207-218

Schmidt, U. and Neugebauer, T. (2007) "Testing Expected Utility in the Presence of Errors" Economic Journal 117, 470-485

Starmer, Ch. and Sugden, R. (1989) "Probability and juxtaposition effects: An experimental investigation of the common ratio effect." Journal of Risk and Uncertainty 2 , $159-178$

Tversky, A. and Kahneman, D. (1986) "Rational choice and the framing of decisions" Journal of Business 59, S251-S278

Tversky, A. and Russo, J. E. (1969) "Substitutability and Similarity in Binary Choices" Journal of Mathematical Psychology 6, 1-12

von Neumann, J. and Morgenstern, O. (1944): "Theory of Games and Economic Behavior, " Princeton, Princeton University Press

Wu, G. (1994) "An Empirical Test of Ordinal Independence" Journal of Risk and Uncertainty 9, 39-60

$\mathrm{Wu}$, G. and Gonzalez, R. (1996) "Curvature of the Probability Weighting Function." Management Science 42, 1676-90

Zimmerman, H.J., Gaines, B.R. and Zadeh, L. A. (1984) "Fuzzy Sets and Decision Analysis", North-Holland 


\section{Appendix}

Before we prove Proposition 1, it is convenient to prove the following two lemmas.

Lemma A1 If Axioms 1-4 hold then $P(A, 0.5 A+0.5 B)=0.5$ for any two lotteries $A, B \in \mathscr{L}$ such that $A \neq B$ and $P(A, B)=0.5$.

Axiom 4 implies that $P(A, 0.5 A+0.5 B)=P(0.5 A+0.5 B, B)$. Let us denote this probability by $q$. Suppose first that $q \geq 0.5$. If $P(B, A) \geq 0.5$ and $P(A, 0.5 A+0.5 B) \geq 0.5$ then Axiom $2 \mathrm{a}$ implies that $P(B, 0.5 A+0.5 B) \geq 0.5$. Axiom 1 implies that $P(B, 0.5 A+0.5 B)=1-q$. Since $q \geq 0.5$ and $1-q \geq 0.5$ it must be the case that $q=0.5$.

Similarly, if $q \leq 0.5$ then $P(B, 0.5 A+0.5 B)=1-q \geq 0.5$. If $\quad P(A, B) \geq 0.5 \quad$ and $P(B, 0.5 A+0.5 B) \geq 0.5$ then Axiom $2 \mathrm{a}$ implies that $P(A, 0.5 A+0.5 B) \geq 0.5$. If $1-q \geq 0.5$ and $q \geq 0.5$ it must be the case that $q=0.5$. This completes the proof of Lemma A1.

Lemma A2 If Axioms 1-4 hold then $P(A, \alpha A+(1-\alpha) B) \geq 0.5$ and $P(B, \alpha A+(1-\alpha) B) \leq 0.5$ for any probability $\alpha \in(0,1)$ and any two lotteries $A, B \in \mathscr{L}$ such that $A \neq B$ and $P(A, B) \geq 0.5$.

Proof by contradiction. Suppose there is $\alpha \in(0,1)$ such that $P(A, \alpha A+(1-\alpha) B)<0.5$. We will construct a sequence $\left\{\alpha_{k}\right\}_{k=1}^{\infty}$ such that $\lim _{k \rightarrow \infty} \alpha_{k}=\alpha$ and $P\left(A, \alpha_{k} A+\left(1-\alpha_{k}\right) B\right)=0.5$ for every $k \in \mathbb{N}$. Axiom 3 implies that the sets $\{\beta \in[0, \alpha] \mid P(A, \beta A+(1-\beta) B) \geq 0.5\}$ and $\{\beta \in[0, \alpha] \mid P(A, \beta A+(1-\beta) B) \leq 0.5\}$ are closed. Axiom 1 guarantees that every $\beta \in[0, \alpha]$ belongs to at least one of these two sets. Since both sets are nonempty ( $\beta=0$ belongs to the first set and $\beta=\alpha$ belongs to the second set), there is at least one $\widetilde{\beta}$ that belongs to both sets. This is the first element of our sequence $\alpha_{1}=\widetilde{\beta}$. 
If $P\left(A, \alpha_{1} A+\left(1-\alpha_{1}\right) B\right)=0.5$ then $P\left(A, 0.5\left(1+\alpha_{1}\right) A+0.5\left(1-\alpha_{1}\right) B\right)=0.5 \quad$ due to Lemma A1. The second element of our sequence is $\alpha_{2}=0.5\left(1+\alpha_{1}\right)$. If $\alpha_{2}=\alpha$ then we have an immediate contradiction to our initial assumption that $P(A, \alpha A+(1-\alpha) B)<0.5$. If $\alpha_{2}<\alpha$ then the third element of our sequence is $\alpha_{3}=0.5\left(1+\alpha_{2}\right) .{ }^{9}$ If $\alpha_{2}>\alpha$ then the third element of our sequence is $\alpha_{3}=0.5\left(\alpha_{1}+\alpha_{2}\right) .{ }^{10}$ By continuing in this fashion ad infinitum, we construct sequence $\left\{\alpha_{k}\right\}_{k=1}^{\infty}$ that is recursively defined by $\alpha_{k+1}=\alpha_{k}+\left(1-\alpha_{1}\right) / 2^{k}$ if $a_{k}<\alpha$ and $\alpha_{k+1}=\alpha_{k}-\left(1-\alpha_{1}\right) / 2^{k}$ if $a_{k}>\alpha, k \in \mathbb{N}$.

Axiom 3 implies that the sets $\left\{\beta \in\left[0, \max _{k \in \mathbf{N}}\left\{\alpha_{k}\right\}\right] \mid P(A, \beta A+(1-\beta) B) \geq 0.5\right\}$ and $\left\{\beta \in\left[0, \max _{k \in \mathbf{N}}\left\{\alpha_{k}\right\}\right] \mid P(A, \beta A+(1-\beta) B) \leq 0.5\right\}$ are closed. Each element of the sequence $\left\{\alpha_{k}\right\}_{k=1}^{\infty}$ belongs to both of these sets. Hence, the limit of the sequence $\lim _{k \rightarrow \infty} \alpha_{k}=\alpha$ also belongs to both of these sets. This implies that $P(A, \alpha A+(1-\alpha) B)=0.5$. However, this contradicts to our initial assumption that $P(A, \alpha A+(1-\alpha) B)<0.5$. We arrive at a similar contradiction if we assume that there exists $\alpha \in(0,1)$ such that $P(B, \alpha A+(1-\alpha) B)>0.5$. This completes the proof of Lemma A2.

${ }^{9}$ If $P\left(A, \alpha_{2} A+\left(1-\alpha_{2}\right) B\right)=0.5$ then $P\left(A, 0.5\left(1+\alpha_{2}\right) A+0.5\left(1-\alpha_{2}\right) B\right)=0.5$ due to Lemma A1. ${ }^{10}$ If $P\left(A, \alpha_{1} A+\left(1-\alpha_{1}\right) B\right)=0.5$ and $P\left(A, \alpha_{2} A+\left(1-\alpha_{2}\right) B\right)=0.5$ then Axiom 2a implies that $P\left(\alpha_{1} A+\left(1-\alpha_{1}\right) B, \alpha_{2} A+\left(1-\alpha_{2}\right) B\right)=0.5$. If the latter equality holds then Lemma A1 implies that $P\left(\alpha_{1} A+\left(1-\alpha_{1}\right) B, 0.5\left(\alpha_{1}+\alpha_{2}\right) A+\left(1-0.5\left(\alpha_{1}+\alpha_{2}\right)\right) B\right)=0.5$. If $P\left(A, \alpha_{1} A+\left(1-\alpha_{1}\right) B\right)=0.5$ and $P\left(\alpha_{1} A+\left(1-\alpha_{1}\right) B, 0.5\left(\alpha_{1}+\alpha_{2}\right) A+\left(1-0.5\left(\alpha_{1}+\alpha_{2}\right)\right) B\right)=0.5$ then Axiom 2a implies that $P\left(A, 0.5\left(\alpha_{1}+\alpha_{2}\right) A+\left(1-0.5\left(\alpha_{1}+\alpha_{2}\right)\right) B\right)=0.5$. 


\section{Proof of Proposition 1}

Since $X$ is a finite set, Axioms 1 and 2a imply that the elements of $X$ can be ordered so that $P\left(X_{i}, X_{j}\right) \geq 0.5$ if $i>j$, for any $i, j \in\{1, \ldots, n\}$. We will now prove Proposition 1 in several steps.

Step 1. Prove that $P\left(X_{n}, A\right) \geq 0.5$ and $P\left(X_{1}, A\right) \leq 0.5$ for any $A\left(a_{1}, \ldots, a_{n}\right) \in \mathscr{L}$ such that $A \neq X_{1}$ and $A \neq X_{n}$.

Proof by mathematical induction. If $n=2$ then Lemma A2 immediately implies that $P\left(X_{2}, a_{2} X_{2}+\left(1-a_{2}\right) X_{1}\right) \geq 0.5$ and $P\left(X_{1}, a_{2} X_{2}+\left(1-a_{2}\right) X_{1}\right) \leq 0.5$ for any probability $a_{2} \in(0,1)$. Let us now assume that the statement holds for any $n \leq k-1$ and let us prove that it also holds for $n=k$. Note that lottery $A\left(a_{1}, \ldots, a_{k}\right)$ can be written as a compound lottery $a_{k} X_{k}+\left(1-a_{k}\right) A^{\prime}$, where lottery $A^{\prime}$ is $\left(a_{1} /\left(1-a_{k}\right), \ldots, a_{k-1} /\left(1-a_{k}\right), 0\right)$. Since $P\left(X_{k-1}, A^{\prime}\right) \geq 0.5$ due to the assumption of mathematical induction and $P\left(X_{k}, X_{k-1}\right) \geq 0.5$ because of the assumed ordering of outcomes, Axiom 2a implies that $P\left(X_{k}, A^{\prime}\right) \geq 0.5$. Lemma A2 then implies that $P\left(X_{k}, a_{k} X_{k}+\left(1-a_{k}\right) A^{\prime}\right) \geq 0.5$ for any $a_{k} \in(0,1)$. Similarly, we can show that $P\left(X_{1}, A\right) \leq 0.5$ if lottery $A\left(a_{1}, \ldots, a_{k}\right)$ is written as a compound lottery $a_{1} X_{1}+\left(1-a_{1}\right) A^{\prime \prime}$, where lottery $A^{\prime \prime}$ is $\left(0, a_{2} /\left(1-a_{1}\right), \ldots, a_{k} /\left(1-a_{1}\right)\right)$.

Step 2. Prove Proposition 1 in case when $P\left(X_{n}, X_{1}\right)=0.5$.

In step 1 we already established that $P\left(X_{n}, A\right) \geq 0.5$ for any $A \in \mathscr{L}, A \neq X_{n}$. Since $P\left(X_{1}, X_{n}\right)=0.5$ and $P\left(X_{n}, A\right) \geq 0.5$, Axiom 2a implies that $P\left(X_{1}, A\right) \geq 0.5$. However, we

already established that $P\left(X_{1}, A\right) \leq 0.5$ in step 1 . Therefore, it must be the case that 
$P\left(X_{1}, A\right)=0.5$ for any $A \in \mathscr{L}, A \neq X_{1}$. If $P\left(A, X_{1}\right)=0.5$ and $P\left(X_{1}, B\right)=0.5$ for any $A, B \in \mathscr{L}$, $A, B \neq X_{1}$, Axiom 2a implies that $P(A, B)=0.5$ for any $A, B \in \mathscr{L}, A \neq B$. These degenerate choice probabilities can be represented by assignment $u_{i}=0, i \in\{1, \ldots, n\}$. Therefore, we will now consider only the case when $P\left(X_{n}, X_{1}\right)>0.5$.

Step 3. Prove that for any $A \in \mathscr{L}$ such that $A \neq \alpha X_{n}+(1-\alpha) X_{1}$ for some $\alpha \in[0,1]$, there is a unique $\alpha_{A} \in[0,1]$ such that $P\left(A, \alpha_{A} X_{n}+\left(1-\alpha_{A}\right) X_{1}\right)=0.5$.

Axiom 3 implies that the sets $\left\{\alpha \in[0,1] \mid P\left(A, \alpha X_{n}+(1-\alpha) X_{1}\right) \geq 0.5\right\}$ and $\left\{\alpha \in[0,1] \mid P\left(A, \alpha X_{n}+(1-\alpha) X_{1}\right) \leq 0.5\right\}$ are closed. Axiom 1 guarantees that every $\alpha \in[0,1]$ belongs to at least one of these two sets. Since both sets are nonempty ( $\alpha=0$ belongs to the first set and $\alpha=1$ belongs to the second set), there is at least one $\alpha_{A} \in[0,1]$ that belongs to both sets. Let us now prove that $\alpha_{A}$ is unique.

Suppose there is $\beta_{A} \neq \alpha_{A}$ such that $P\left(A, \beta_{A} X_{n}+\left(1-\beta_{A}\right) X_{1}\right)=0.5$. Without loss of generality, let us assume that $\beta_{A}>\alpha_{A}$ and let us denote the difference $\beta_{A}-\alpha_{A}$ with $\delta$. If $P\left(\beta_{A} X_{n}+\left(1-\beta_{A}\right) X_{1}, A\right)=0.5$ and $P\left(A, \alpha_{A} X_{n}+\left(1-\alpha_{A}\right) X_{1}\right)=0.5$ then Axiom 2a implies that $P\left(\beta_{A} X_{n}+\left(1-\beta_{A}\right) X_{1}, \alpha_{A} X_{n}+\left(1-\alpha_{A}\right) X_{1}\right)=0.5$. Axiom 4 then immediately implies that $\quad P\left(\beta_{A} X_{n}+\left(1-\beta_{A}\right) X_{1}, \alpha_{A} X_{n}+\left(1-\alpha_{A}\right) X_{1}\right)=P\left(\delta X_{n}+(1-\delta) X_{1}, X_{1}\right)=0.5 . \quad$ If $P\left(\delta X_{n}+(1-\delta) X_{1}, X_{1}\right)=0.5$ then $P\left(2 \delta X_{n}+(1-2 \delta) X_{1}, \delta X_{n}+(1-\delta) X_{1}\right)=0.5$ due to Axiom 4 and $\operatorname{Pr}\left(2 \delta X_{n}+(1-2 \delta) X_{1}, X_{1}\right)=0.5$ due to Axiom 2a. Applying this reasoning $k=\operatorname{int}\left\{\beta_{A} / \delta\right\}$ times we obtain that $P\left(k \delta X_{n}+(1-k \delta) X_{1}, X_{1}\right)=0.5$. 
Similarly, Axiom 4 implies that $P\left(\beta_{A} X_{n}+\left(1-\beta_{A}\right) X_{1}, \alpha_{A} X_{n}+\left(1-\alpha_{A}\right) X_{1}\right)=$ $=P\left(X_{n},(1-\delta) X_{n}+\delta X_{1}\right)=0.5$. If $P\left(X_{n},(1-\delta) X_{n}+\delta X_{1}\right)=0.5$ then Axiom 4 implies that $P\left((1-\delta) X_{n}+\delta X_{1},(1-2 \delta) X_{n}+2 \delta X_{1}\right)=0.5$ and Axiom 2a subsequently implies that $P\left(X_{n},(1-2 \delta) X_{n}+2 \delta X_{1}\right)=0.5$. Applying this reasoning $m=\operatorname{int}\left\{\left(1-\alpha_{A}\right) / \delta\right\}$ times we obtain that $P\left(X_{n},(1-m \delta) X_{n}+m \delta X_{1}\right)=0.5$. If lottery $(1-m \delta) X_{n}+m \delta X_{1}$ coincides with lottery $k \delta X_{n}+(1-k \delta) X_{1}$ then Axiom 2a implies that $P\left(X_{n}, X_{1}\right)=0.5$. However, we already considered this case in step 2. Thus, let us consider the case when $(1-m \delta) X_{n}+m \delta X_{1}$ is not the same lottery as $k \delta X_{n}+(1-k \delta) X_{1}$.

Since $P\left(\beta_{A} X_{n}+\left(1-\beta_{A}\right) X_{1}, \alpha_{A} X_{n}+\left(1-\alpha_{A}\right) X_{1}\right)=0.5$ Lemma A2 implies that $P\left(\beta_{A} X_{n}+\left(1-\beta_{A}\right) X_{1}, \alpha X_{n}+(1-\alpha) X_{1}\right)=0.5$ for any $\alpha \in\left(\alpha_{A}, \beta_{A}\right)$. Axiom 2a then implies that $P\left(\alpha X_{n}+(1-\alpha) X_{1}, \beta X_{n}+(1-\beta) X_{1}\right)=0.5$ for any $\alpha, \beta \in\left[\alpha_{A}, \beta_{A}\right], \quad \alpha \neq \beta$. Since $k \delta \in\left[\alpha_{A}, \beta_{A}\right]$ and $1-m \delta \in\left[\alpha_{A}, \beta_{A}\right]$ by our choice of integers $k$ and $m$ it follows that $P\left((1-m \delta) X_{n}+m \delta X_{1}, k \delta X_{n}+(1-k \delta) X_{1}\right)=0.5$. Since we already established that $P\left(X_{n},(1-m \delta) X_{n}+m \delta X_{1}\right)=0.5$ and $P\left(k \delta X_{n}+(1-k \delta) X_{1}, X_{1}\right)=0.5$, Axiom 2a then implies that $P\left(X_{n}, X_{1}\right)=0.5$. However, we already considered this case in step 2 . Therefore, it must be the case that $\beta_{L}=\alpha_{L}$.

Step 4. Prove that for any $A \in \mathscr{L}$ there is a unique number $U(A)$ such that $P(A, B)=0.5$ if and only if $U(A)=U(B)$ for any $B \in \mathscr{L}, A \neq B$.

Let us assign a number $U(A)$ to any lottery $A \in \mathscr{L}$ in the following manner. If $A=\alpha X_{n}+(1-\alpha) X_{1}$ for some $\alpha \in[0,1]$ then let $U(A)=\alpha$. If $A \neq \alpha X_{n}+(1-\alpha) X_{1}$ for any 
$\alpha \in[0,1]$ then there is a unique $\alpha_{A} \in[0,1]$ such that $P\left(A, \alpha_{A} X_{n}+\left(1-\alpha_{A}\right) X_{1}\right)=0.5$ by step 3 and let $U(A)=\alpha_{A}$. Let us now prove that such assignment meets our goal. If $P(A, B)=0.5$ and $A=U(A) X_{n}+(1-U(A)) X_{1}$ then $U(A)=U(B)$ because we proved in step 3 that there is only one $\alpha_{B} \in[0,1]$ such that $P\left(B, \alpha_{B} X_{n}+\left(1-\alpha_{B}\right) X_{1}\right)=0.5$. If $P(A, B)=0.5$ and $A \neq U(A) X_{n}+(1-U(A)) X_{1}$ then $P\left(A, U(A) X_{n}+(1-U(A)) X_{1}\right)=0.5$. In this case if $B=U(B) X_{n}+(1-U(B)) X_{1}$, then it must be the case that $U(A)=U(B)$ because we proved in step 3 that there is only one $\alpha_{A} \in[0,1]$ such that $P\left(A, \alpha_{A} X_{n}+\left(1-\alpha_{A}\right) X_{1}\right)=0.5$. If $B \neq U(B) X_{n}+(1-U(B)) X_{1}$, then Axiom 2a implies that $P\left(B, U(A) X_{n}+(1-U(A)) X_{1}\right)=0.5$ and again it must be the case that $U(A)=U(B)$.

If $\quad U(A)=U(B)$ and $A=U(A) X_{n}+(1-U(A)) X_{1} \quad$ then we can write $P\left(B, U(B) X_{n}+(1-U(B)) X_{1}\right)=P(B, A)=0.5$. If $U(A)=U(B)$ and $A \neq U(A) X_{n}+(1-U(A)) X_{1}$ then we can write $P\left(A, U(A) X_{n}+(1-U(A)) X_{1}\right)=P\left(A, U(B) X_{n}+(1-U(B)) X_{1}\right)=0.5$. In this case if $B=U(B) X_{n}+(1-U(B)) X_{1}$ then we immediately have $P(A, B)=0.5$. If $B \neq U(B) X_{n}+(1-U(B)) X_{1} \quad$ then $\quad$ we have $P\left(B, U(B) X_{n}+(1-U(B)) X_{1}\right)=0.5 \quad$ and $P\left(A, U(B) X_{n}+(1-U(B)) X_{1}\right)=0.5$. Axiom 2a then implies that $P(A, B)=0.5$.

Step 5. Prove that $U(\alpha A+(1-\alpha) B)=\alpha U(A)+(1-\alpha) U(B)$ for any $A, B \in \mathscr{L}$ and any $\alpha \in[0,1]$.

If $A=U(A) X_{n}+(1-U(A)) X_{1}$ and $B=U(B) X_{n}+(1-U(B)) X_{1}$ then $\alpha A+(1-\alpha) B=$ $=\alpha\left[U(A) X_{n}+(1-U(A)) X_{1}\right]+(1-\alpha)\left[U(B) X_{n}+(1-U(B)) X_{1}\right]=[\alpha U(A)+(1-\alpha) U(B)] X_{n}+$ $+[1-\alpha U(A)-(1-\alpha) U(B)] X_{1}$. Thus, we have $U(\alpha A+(1-\alpha) B)=\alpha U(A)+(1-\alpha) U(B)$. If 
$A \neq U(A) X_{n}+(1-U(A)) X_{1}$ then we have $P\left(A, U(A) X_{n}+(1-U(A)) X_{1}\right)=0.5$ and Lemma A2 implies that $P\left(\alpha A+(1-\alpha)\left[U(A) X_{n}+(1-U(A)) X_{1}\right], U(A) X_{n}+(1-U(A)) X_{1}\right)=0.5$. Axiom 4 then implies $P\left(\alpha A+(1-\alpha)\left[U(B) X_{n}+(1-U(B)) X_{1}\right], \alpha\left[U(A) X_{n}+(1-U(A)) X_{1}\right]+\right.$ $\left.+(1-\alpha)\left[U(B) X_{n}+(1-U(B)) X_{1}\right]\right)=0.5$, which can be rearranged into the following equation: $P\left(\alpha A+(1-\alpha)\left[U(B) X_{n}+(1-U(B)) X_{1}\right][\alpha U(A)+(1-\alpha) U(B)] X_{n}+\right.$ $\left.+[1-\alpha U(A)-(1-\alpha) U(B)] X_{1}\right)=0.5$. If $B=U(B) X_{n}+(1-U(B)) X_{1}$ we immediately have $U(\alpha A+(1-\alpha) B)=\alpha U(A)+(1-\alpha) U(B)$

If $B \neq U(B) X_{n}+(1-U(B)) X_{1}$ then $P\left(B, U(B) X_{n}+(1-U(B)) X_{1}\right)=0.5$ and Lemma A2 implies that $P\left(\alpha\left[U(B) X_{n}+(1-U(B)) X_{1}\right]+(1-\alpha) B, U(B) X_{n}+(1-U(B)) X_{1}\right)=0.5$. Axiom 4 then implies $P\left(\alpha A+(1-\alpha) B, \alpha A+(1-\alpha)\left[U(B) X_{n}+(1-U(B)) X_{1}\right]\right)=0.5$. Axiom 2a then implies that $P\left(\alpha A+(1-\alpha) B,[\alpha U(A)+(1-\alpha) U(B)] X_{n}+[1-\alpha U(A)-(1-\alpha) U(B)] X_{1}\right)=0.5$. Thus we have again $U(\alpha A+(1-\alpha) B)=\alpha U(A)+(1-\alpha) U(B)$.

Hence, if we set $u_{i}=U\left(X_{i}\right)$ then $P(A, B)=0.5$ if and only if $\sum_{i=1}^{n} u_{i} a_{i}=\sum_{i=1}^{n} u_{i} b_{i}$ for any two lotteries $A\left(a_{1}, \ldots, a_{n}\right), B\left(b_{1}, \ldots, b_{n}\right) \in \mathscr{L}, A \neq B$. Q.E.D.

\section{Proof of Proposition 2}

Proof by mathematical induction. Let us first prove Proposition 2 for lotteries with only two outcomes. In this case lottery $A \equiv a_{1} X_{1}+\left(1-a_{1}\right) X_{2}$ weakly dominates lottery $B \equiv b_{1} X_{1}+\left(1-b_{1}\right) X_{2}$ if $a_{1} \leq b_{1}$. Since $A \neq B$, it must be that $a_{1}<b_{1}$. Axiom 4 implies that $P(A, B)=P\left(a_{1} X_{1}+\left(1-a_{1}\right) X_{2}, b_{1} X_{1}+\left(1-b_{1}\right) X_{2}\right)=P\left(X_{2},\left(b_{1}-a_{1}\right) X_{1}+\left(1-b_{1}+a_{1}\right) X_{2}\right)$. 
Axiom 5 implies that $P\left(X_{2},\left(b_{1}-a_{1}\right) X_{1}+\left(1-b_{1}+a_{1}\right) X_{2}\right) \in\{0,1\}$. Let us now prove that this probability cannot be equal to zero. We will construct a sequence $\left\{\alpha_{k}\right\}_{k=1}^{\infty}$ such that $\lim _{k \rightarrow \infty} \alpha_{k}=b_{1}-a_{1}$ and $P\left(X_{2}, \alpha_{k} X_{1}+\left(1-\alpha_{k}\right) X_{2}\right)=1$ for every $k \in \mathbb{N}$. The first element of our sequence is $\alpha_{1}=0.5$. Let us prove that $P\left(X_{2}, 0.5 X_{1}+0.5 X_{2}\right)=1$. Axiom 5 states that $P\left(X_{2}, 0.5 X_{1}+0.5 X_{2}\right)$ is either zero or one. If $P\left(X_{2}, 0.5 X_{1}+0.5 X_{2}\right)=0$ then Axiom 1 implies that $P\left(0.5 X_{1}+0.5 X_{2}, X_{2}\right)=1$. If $P\left(0.5 X_{1}+0.5 X_{2}, X_{2}\right)=1$ then Axiom 4 implies that $P\left(X_{1}, 0.5 X_{1}+0.5 X_{2}\right)=1$. If $P\left(X_{2}, X_{1}\right)=1$ and $P\left(X_{1}, 0.5 X_{1}+0.5 X_{2}\right)=1$ then Axiom $2 \mathrm{~b}$ implies that $P\left(X_{2}, 0.5 X_{1}+0.5 X_{2}\right)=1$, which contradicts to our initial assumption that $P\left(X_{2}, 0.5 X_{1}+0.5 X_{2}\right)=0$. Therefore, it must be the case that $P\left(X_{2}, 0.5 X_{1}+0.5 X_{2}\right)=1$.

If $b_{1}-a_{1}=0.5$ then we immediately have our result that $P(A, B)$ cannot be zero. If $b_{1}-a_{1}>0.5$ then the second element of our sequence is $\alpha_{2}=0.75$. If $b_{1}-a_{1}<0.5$ then the second element of our sequence is $\alpha_{2}=0.25$. Note that $P\left(X_{2}, \alpha_{2} X_{1}+\left(1-\alpha_{2}\right) X_{2}\right)=1$ by a similar argument to the one that we established in the previous paragraph for $\alpha_{1}=0.5$. By continuing in this fashion ad infinitum, we end up with a sequence $\left\{\alpha_{k}\right\}_{k=1}^{\infty}$ that is recursively defined by $\alpha_{k+1}=\alpha_{k}+1 / 2^{k+1}$ if $a_{k}<b_{1}-a_{1}$ and $\alpha_{k+1}=\alpha_{k}-1 / 2^{k+1}$ if $a_{k}>b_{1}-a_{1}, k \in \mathbb{N}$.

Axiom 3 implies that the set $\left\{\beta \in\left[\min _{k \in \mathbf{N}}\left\{\alpha_{k}\right\}, 1\right] \mid P\left(X_{2}, \beta X_{1}+(1-\beta) X_{2}\right) \geq 0.5\right\}$ is closed. Each element of the sequence $\left\{\alpha_{k}\right\}_{k=1}^{\infty}$ belongs to this set. Thus, the limit of the sequence should be in this set as well. This means that $P\left(X_{2},\left(b_{1}-a_{1}\right) X_{1}+\left(1-b_{1}+a_{1}\right) X_{2}\right) \geq 0.5$. 
Thus, this probability cannot be equal to zero and it must be equal to one due to Axiom 5 . Therefore, we can conclude that $P(A, B)=P\left(X_{2},\left(b_{1}-a_{1}\right) X_{1}+\left(1-b_{1}+a_{1}\right) X_{2}\right)=1$.

Suppose now that Proposition 2 holds for any lotteries with $n-1$ or less outcomes and let us prove that it also holds for lotteries with $n$ outcomes. If lottery $A\left(a_{1}, \ldots, a_{n}\right)$ weakly dominated lottery $B\left(b_{1}, \ldots, b_{n}\right)$ then $a_{1} \leq b_{1}$ and we can apply Axiom 4 as follows: $P(A, B)=P\left(\left(a_{1}+a_{2}\right) X_{2}+a_{3} X_{3}+\ldots+a_{n} X_{n},\left(b_{1}-a_{1}\right) X_{1}+\left(b_{2}+a_{1}\right) X_{2}+b_{3} X_{3}+\ldots+b_{n} X_{n}\right)$.

Note that lottery $C\left(0, a_{1}+a_{2}, a_{3}, \ldots, a_{n}\right)$ weakly dominated lottery $D\left(0, b_{1}+b_{2}, b_{3}, \ldots, b_{n}\right)$ and $P(C, D)=1$ due to the assumption of mathematical induction. Axiom 4 implies that lottery $D\left(0, b_{1}+b_{2}, b_{3}, \ldots, b_{n}\right)$ is chosen over lottery $F\left(b_{1}-a_{1}, b_{2}+a_{1}, b_{3}, \ldots, b_{n}\right)$ with probability $P(D, F)=P\left(X_{2},\left(b_{1}-a_{1}\right) X_{1}+\left(1-b_{1}+a_{1}\right) X_{2}\right)=1$. If $P(C, D)=1$ and $P(D, F)=1$ then Axiom $2 \mathrm{~b}$ implies that $P(C, F)=1$. Since we already established that $P(A, B)=P(C, F)$, we can conclude that $P(A, B)=1$. Q.E.D.

\section{Proof of Proposition 3}

For any $A\left(a_{1}, \ldots, a_{n}\right), B\left(b_{1}, \ldots, b_{n}\right) \in \mathscr{L}$ we can construct lottery $C$ that yields outcome $x_{i}$ with probability $\min \left\{\sum_{j=1}^{i} a_{j}, \sum_{j=1}^{i} b_{j}\right\}-\min \left\{\sum_{j=1}^{i-1} a_{j}, \sum_{j=1}^{i-1} b_{j}\right\}$ and lottery $D$ that yields outcome $x_{i}$ with probability $\max \left\{\sum_{j=1}^{i} a_{j}, \sum_{j=1}^{i} b_{j}\right\}-\max \left\{\sum_{j=1}^{i-1} a_{j}, \sum_{j=1}^{i-1} b_{j}\right\}, \quad i \in\{1, \ldots, n\}$. Note that $C \stackrel{d}{\gtrsim} A$ and $C \stackrel{d}{\gtrsim} B$. Suppose that there is another lottery $F\left(f_{1}, \ldots, f_{n}\right) \in \mathscr{L}$ such that $F \succsim A$ and $F \succsim B$ and $C \succsim F$. Then it must be the case that $\sum_{i=1}^{j} f_{i} \leq \sum_{i=1}^{j} a_{i}, \sum_{i=1}^{j} f_{i} \leq \sum_{i=1}^{j} b_{i}$ and $\min \left\{\sum_{j=1}^{i} a_{j}, \sum_{j=1}^{i} b_{j}\right\} \leq \sum_{i=1}^{j} f_{i}$ for any $j \in\{1, \ldots, n\}$. These three inequalities imply that 
$\sum_{i=1}^{j} f_{i}=\min \left\{\sum_{j=1}^{i} a_{j}, \sum_{j=1}^{i} b_{j}\right\}$ for any $j \in\{1, \ldots, n\}$ i.e. $F=C$. In other words, lottery $C$ is a least upper bound on $A$ and $B$. Similarly, we can show that lottery $D$ is a greatest lower bound on $A$ and B. Q.E.D.

\section{Proof of Proposition 4}

It is a relatively straightforward algebraic exercise to demonstrate that if binary choice probabilities admit representation (2) then they satisfy Axioms 1-6. Therefore, I will only prove the sufficiency of Axioms 1-6.

Consider an arbitrary pair of lotteries $A, B \in \mathscr{L}$ such that $A \neq B$. Note that if $A=A \wedge B$ then $P(A, B)=0$ due to Proposition 2 and equation (2) holds trivially if we set $\varphi(0)=0$ (in this case $c_{i}=a_{i}$ for all $i \in\{1, \ldots, n\}$ ). Similarly, if $B=A \wedge B$ then $P(A, B)=1$ due to Proposition 2 and equation (2) holds trivially (in this case $c_{i}=b_{i}$ for all $i \in\{1, \ldots, n\}$ ). Therefore, we need to consider only the case when $A \neq A \wedge B$ and $B \neq A \wedge B$.

Let us assume, without loss of generality, that $P(A, B) \leq 1 / 2$. If Axiom 6 holds, then there is a function $f: \mathscr{L} \times \mathscr{L} \times \mathscr{L} \rightarrow \mathbb{R}_{+}$such that

$$
\frac{P(A, B)}{P(B, A)} / \frac{P(A, C)}{P(C, A)}=f(B, C, A \wedge B),
$$

where $C \in \mathscr{L}$ is an arbitrary lottery such that $A \neq C$ and $A \wedge B=A \wedge C$. Let us prove Proposition 4 in several steps.

Step 1. Prove that function $f$ has the following form $f(B, C, A \wedge B)=\frac{\varphi(C, A \wedge B)}{\varphi(B, A \wedge B)}$.

Since equation (10) holds for any $C \in \mathscr{L}$ such that $A \neq C$ and $A \wedge B=A \wedge C$ we can write it for another lottery $D \in \mathscr{L}$ such that $A \neq D$ and $A \wedge B=A \wedge D$ : 


$$
\frac{P(A, B)}{P(B, A)} / \frac{P(A, D)}{P(D, A)}=f(B, D, A \wedge B)
$$

We can also substitute lottery $B$ with lottery $D$ in equation (10) and obtain:

$$
\frac{P(A, D)}{P(D, A)} / \frac{P(A, C)}{P(C, A)}=f(D, C, A \wedge B) .
$$

If we multiply equation (11) on equation (12) and use the identity in equation (10), we obtain:

$$
f(B, C, A \wedge B)=f(B, D, A \wedge B) \cdot f(D, C, A \wedge B) .
$$

Equation (13) is a generalized multiplicative Sincov functional equation (e.g. Aczél, 1966). Function $f\left(\right.$.) is a solution to (13) if and only if there exists a function $\varphi: \mathscr{L} \times \mathscr{L} \rightarrow \mathbb{R}_{+}$ such that $f(B, C, A \wedge B)=\varphi(C, A \wedge B) / \varphi(B, A \wedge B)$.

If Axioms 1-4 hold then Proposition 1 implies that there is an assignment of real numbers $u_{i}$ to every outcome $x_{i}, i \in\{1, \ldots, n\}$, such that $P(A, B)=0.5$ if and only if $\sum_{i=1}^{n} u_{i} a_{i}=\sum_{i=1}^{n} u_{i} b_{i}$ for any two lotteries $A\left(a_{1}, \ldots, a_{n}\right), B\left(b_{1}, \ldots, b_{n}\right) \in \mathcal{L}, \quad A \neq B$. Let function $U: \mathcal{L} \rightarrow \mathbb{R}$ be the expected utility function i.e. $U(A) \equiv \sum_{i=1}^{n} u_{i} a_{i}$ for any $A\left(a_{1}, \ldots, a_{n}\right) \in \mathscr{L}$.

Step 2. Prove that function $\varphi$ has the following form $\varphi(A, A \wedge B)=\varphi(U(A), A \wedge B)$.

Axiom 3 implies that the sets $\{\alpha \in[0,1] \mid P(A, \alpha B+(1-\alpha)(A \wedge B)) \geq 0.5\}$ and $\{\alpha \in[0,1] \mid P(A, \alpha B+(1-\alpha)(A \wedge B)) \leq 0.5\}$ are closed. Both sets are non-empty $(\alpha=0$ belongs to the first set due to Proposition 2 and $\alpha=1$ belongs to the second set, because we assumed that $P(A, B) \leq 1 / 2)$. Hence, there is $\alpha_{A} \in[0,1]$ that belongs to both sets i.e. 
$P\left(A, \alpha_{A} B+\left(1-\alpha_{A}\right)(A \wedge B)\right)=0.5$. According to Proposition 1 the latter equality holds if and only if $U\left(\alpha_{A} B+\left(1-\alpha_{A}\right)(A \wedge B)\right)=U(A)$. This implies that probability $\alpha_{A}$ is unique and it is equal to $(U(A)-U(A \wedge B)) /(U(B)-U(A \wedge B))$. Let $F \equiv \alpha_{A} B+\left(1-\alpha_{A}\right) A \wedge B$.

Note that $A \wedge F=A \wedge\left[\alpha_{A} B+\left(1-\alpha_{A}\right) A \wedge B\right]=A \wedge B$ and $P(A, F)=P(F, A)=0.5$ due to Axiom 1. Thus, we can substitute lottery $C$ with lottery $F$ in equation $(10)$ and obtain:

$$
\frac{P(A, B)}{P(B, A)} / \frac{0.5}{0.5}=\frac{\varphi(F, A \wedge B)}{\varphi(B, A \wedge B)} .
$$

Equation (14) holds for any $A, B \in \mathscr{L}$ such that $A \neq B \neq A \wedge B$ and a lottery $F \in \mathscr{L}$ such that $A \wedge F=A \wedge B$ and $U(F)=U(A)$. Since lottery $F$ is unique, we can write $U(F)$ instead of $F$ in the right hand side of (14). Moreover, since $U(F)=U(A)$, we can write just $U(A)$ in the right hand side of (14), which gives the following result:

$$
\frac{P(A, B)}{P(B, A)}=\frac{\varphi(U(A), A \wedge B)}{\varphi(B, A \wedge B)} .
$$

By a symmetry argument we can conclude that $\varphi(B, A \wedge B)=\varphi(U(B), A \wedge B)$. Axiom 1 implies that $P(B, A)=1-P(A, B)$. Plugging this result into (15), we obtain:

$$
P(A, B)=\frac{\varphi(U(A), A \wedge B)}{\varphi(U(A), A \wedge B)+\varphi(U(B), A \wedge B)} .
$$

Step 3. Prove that function $\varphi$ has the form $\varphi(U(A), A \wedge B)=\varphi(U(A)-U(A \wedge B))$.

First of all, since lottery $A \wedge B$ is unique for all $A, B \in \mathscr{L}$ we can write $U(A \wedge B)$ instead of $A \wedge B$ in the right-hand side of (16). Axiom 4 implies that for any lotteries $A, B, C \in \mathscr{L}, A \neq B$, the choice probability $P(0.5 A+0.5 C, 0.5 B+0.5 C)$ does not depend on $C$. 
Note that $[0.5 A+0.5 C] \wedge[0.5 B+0.5 C]=0.5(A \wedge B)+0.5 C$. Using equation (16) we can write:

$$
\begin{aligned}
& P(0.5 A+0.5 C, 0.5 B+0.5 C)= \\
& =\frac{\varphi\left(\frac{1}{2} U(A)+\frac{1}{2} U(C), \frac{1}{2} U(A \wedge B)+\frac{1}{2} U(C)\right)}{\varphi\left(\frac{1}{2} U(A)+\frac{1}{2} U(C), \frac{1}{2} U(A \wedge B)+\frac{1}{2} U(C)\right)+\varphi\left(\frac{1}{2} U(B)+\frac{1}{2} U(C), \frac{1}{2} U(A \wedge B)+\frac{1}{2} U(C)\right)} .
\end{aligned}
$$

Since the right hand side of (17) does not depend on $C$ it must be the case that $\varphi(0.5 U(A)+0.5 U(C), 0.5 U(A \wedge B)+0.5 U(C))$ is independent of $C$. Therefore, we can set $U(C)$ to an arbitrary value e.g. $U(C)=-U(A \wedge B)$. This implies that we can write function $\varphi$ as a function of only one variable $\varphi(U(A), U(A \wedge B))=\varphi(U(A)-U(A \wedge B))$.

To summarize, we have shown that if Axioms 1-6 hold then for any lotteries $A, B \in \mathscr{L}$ such that $A \neq B$ we can write

$$
P(A, B)=\frac{\varphi(U(A)-U(A \wedge B))}{\varphi(U(A)-U(A \wedge B))+\varphi(U(B)-U(A \wedge B))} .
$$

In the proof of Proposition 3 we already established that lottery $A \wedge B$ yields outcome $x_{i}$ with probability $c_{i}=\max \left\{\sum_{j=1}^{i} a_{j}, \sum_{j=1}^{i} b_{j}\right\}-\max \left\{\sum_{j=1}^{i-1} a_{j}, \sum_{j=1}^{i-1} b_{j}\right\}, i \in\{1, \ldots, n\}$. Note that Proposition 1 implies that $P(A, B) \geq 0.5$ if and only if $U(A) \geq U(B)$. Equation (18) implies that $P(A, B) \geq 0.5$ if and only if $\varphi(U(A)-U(A \wedge B)) \geq \varphi(U(B)-U(A \wedge B))$. Thus, if $U(A)-U(A \wedge B) \geq U(B)-U(A \wedge B)$ then $\varphi(U(A)-U(A \wedge B)) \geq \varphi(U(B)-U(A \wedge B))$ i.e. function $\varphi($.$) is non-decreasing. Note that U(A) \geq U(A \wedge B)$ for any $A, B \in \mathscr{L}$ and hence function $\varphi($.$) is on \mathbb{R}_{+}$. Since we initially set $\varphi(0)=0$ and function $\varphi($.$) is non-decreasing, it$ must be the case that $\varphi: \mathbb{R}_{+} \rightarrow \mathbb{R}_{+}$. Q.E.D. 